\title{
Consulta de enfermagem em puericultura na estratégia saúde da família: revisão integrativa
}

RESUMO | Objetivo: analisar a produção científica sobre consulta de puericultura da enfermagem na Estratégia Saúde da Família. Métodos: revisão integrativa da literatura. Foram consultadas as fontes de dados do período 2008 a 2017, com um levantamento de produções pelos descritores enfermagem, puericultura e atenção básica nas Bases de Dados: LILACS, SciELO e MEDLINE. Na etapa inicial da pesquisa encontrou-se 33 artigos. Após aplicar os filtros ano de publicação, disponibilidade, idioma e leitura dos títulos, a amostra totalizou 14 artigos científicos. Resultados: Os atendimentos de enfermagem à saúde da criança acontecem de forma fragmentada, com foco em dados antropométricos, estado nutricional e, por vezes, a imunização. Conclusão: Assim, averiguou-se a necessidade de os gestores promoverem a capacitação dos profissionais de saúde, a criação de um instrumento de padronização do atendimento ao infante aprimorando a sistematização de enfermagem. O estudo limita-se por ter realizado recorte temporal das publicações e por não ter abrangido todas as bases de dados existentes.

Palavras-chaves: Enfermagem; Puericultura; Atenção básica.

ABSTRACT | Objective: to analyze the scientific production about nursing child care consultation in the Family Health Strategy. Methods: integrative literature review. Data sources were consulted from 2008 to 2017, with a survey of production by the descriptors nursing, child care and basic care in the Databases: LILACS, SCIELO and MEDLINE. In the initial stage of the research 33 articles were found. After applying the filters year of publication, availability, language and reading titles, the sample totaled 14 scientific articles. Results: Nursing care for children occurs in a fragmented way, focusing on anthropometric data, nutritional status and, sometimes, immunization. Conclusion: Thus, it was verified the need for managers to promote the qualification of health professionals, the creation of an instrument to standardize infant care, improving nursing systematization. The study is limited by the time-cut of the publications and by not having covered all the existing databases.

Keywords: Nursing; Child Care; Basic attention.

RESUMEN | Objetivo: analizar la producción científica sobre consulta de puericultura de la enfermería en la Estrategia Salud de la Familia. Métodos: revisión integrativa de la literatura. Se consultaron las fuentes de datos del período 2008 a 2017, con un levantamiento de producciones por los descriptores enfermería, puericultura y atención básica en las Bases de Datos: LILACS, SCIELO y MEDLINE. En la etapa inicial de la investigación se encontraron 33 artículos. Después de aplicar los filtros año de publicación, disponibilidad, idioma y lectura de los títulos, la muestra totalizó 14 artículos científicos. Resultados: Las atenciones de enfermería a la salud del niño ocurren de forma fragmentada, con foco en datos antropométricos, estado nutricional y, a veces, la inmunización. Conclusión: Así, se averiguó la necesidad de que los gestores promover la capacitación de los profesionales de salud, la creación de un instrumento de estandarización de la atención al infante mejorando la sistematización de enfermería. El estudio se limita por haber realizado recorte temporal de las publicaciones y por no haber abarcado todas las bases de datos existentes.

Palabras claves: enfermería; cuidado de los niños; Atención básica.

\section{Maria Niná Morais Tavares}

Enfermeira pela Universidade Regional do Cariri (URCA).

\section{José Adelmo da Silva Filho}

Enfermeiro. Especialista em Saúde Mental Coletiva. Mestrando em Enfermagem pela Universidade Regional do Cariri (URCA).

\section{Cicero Rafael Lopes da Silva}

Enfermeiro pela Faculdade de Juazeiro do Norte (FJN).

Antonio Germane Alves Pinto
Enfermeiro. Doutor em Saúde Coleti-
va pela Universidade Estadual do Ceará
(UECE).


INTRODUÇÃO

mortalidade infantil no Bra-
sil em 2016 apresentou
aumento significativo comparado ao ano anterior. Dados do DATASUS mostram que de 2015 para 2016 o coeficiente de mortalidade infantil aumentou em 2,4\%, sendo 12,7 óbitos por 100 nascidos vivos ${ }^{(1)}$.

Frente às necessidades de saúde apresentadas pela população, foram formuladas e colocadas em vigor várias políticas e programas em saúde, dentre as quais evidencia-se a mortalidade materno-infantil que impulsionou a criação de estratégias para diminuir a ocorrência de mortes evitáveis em menores de 02 anos, como o Programa de Acompanhamento do Desenvolvimento e Crescimento Infantil (PADCI), formulado pelo Ministério da Saúde (MS) em $1984^{(3,4,5)}$.

$\mathrm{Na}$ Estratégia Saúde da Família (ESF), a enfermeira é essencial para realizar cuidado integrado à criança, pois desenvolve vínculo com a família e a comunidade promovendo cuidado sistematizado com foco na vigilância do processo de crescimento e desenvolvimento, prevenção de agravos, promoção e manutenção da saúde ${ }^{(6)}$.

Desta forma as atribuições da enfermeira em consulta de puericultura na ESF são: orientar, treinar e definir funções para a equipe de enfermagem; supervisionar as atividades; realizar visitas domiciliares às crianças de risco; desenvolver ações educativas fornecendo informações às mães e aos cuidadores; identificar a situação de saúde da criança relacionando-a as suas condições de vida e considerando suas características biopsicossociais; exercer as atribuições que lhe são conferidas pela Política Nacional da Atenção Básica $(\mathrm{PNAB})^{(6,7)}$.

As ações de intervenção promovem mudanças individuais e coletivas, repercutindo em mudanças de indicadores. Porém, ressalta-se que há lacunas na realização das consultas de puericultura, dentre elas a falta de conhecimento tanto de profissionais como de pais \responsáveis sobre a importância de acompanhar o crescimento e desenvolvimento infantil; a falta de acompanhamento da situação vacinal pela enfermeira; além da dificuldade de implementação de um cuidado sistematizado. Desta forma, a consulta tem foco curativista, enquanto que deveria ter foco preventivo ${ }^{(8)}$.

Diante do exposto, verifica-se a importância do acompanhamento do desenvolvimento e crescimento da criança na Unidade Básica de Saúde (UBS) pelo (a) enfermeiro (a). Como uma importante ferramenta de combate aos agravos evitáveis que acometem tal faixa etária da população, atuando diretamente nos índices de morbidade e mortalidade infantil. Propõe-se o seguinte questionamento: Quais as principais barreiras encontradas pela enfermeira para realização das consultas de puericultura?

Dessa forma, o estudo tem por objetivo analisar a produção científica sobre as barreiras enfrentadas na consulta de puericultura pela enfermeira na Estratégia Saúde da Família.

\section{MÉTODO}

Trata-se de uma revisão integrativa da literatura realizada no mês de maio de 2018. O processo de construção obedeceu às seis etapas utilizadas na elaboração desse tipo de revisão, sendo: identificação do tema e elaboração da pergunta norteadora, estabelecimento de critérios para inclusão e exclusão de estudos, definição das informações a serem extraídas dos estudos selecionados, interpretação/discussão dos resultados, apresentação da revisão/síntese do conhecimento. A revisão integrativa é um método de pesquisa que proporciona a síntese de um conhecimento e incorpora seus resultados significativos na prática ${ }^{(9)}$.

Utilizou-se das seguintes bases de dados: Literatura Latino-Americana e do Caribe em Ciências da Saúde (LILA-
CS), Scientific Electronic Library Online (SciELO) e Literatura Internacional em Ciências da Saúde (MEDLINE). A utilização da LILACS, SciELO e MEDLINE deu-se em virtude de conter textos completos e serviços de fornecimento de cópias de documentos, bem como pela multiplicidade de trabalhos nelas encontrados, dado às diferentes revistas acadêmicas Nacionais e Internacionais que as alimentam. Para melhor efetividade na coleta de dados foram utilizados descritores indicados na DeCS, a saber, Enfermagem, puericultura e atenção básica, combinadas com o operador booleano AND.

Os critérios de inclusão no estudo foram artigos publicados nos últimos 5 anos, no idioma português, que se encontrava disponível na íntegra nas bases de dados e apresentasse afinidade com a temática. Os critérios de exclusão foram os artigos parcialmente publicados, arquivos repetidos, e também aqueles que se encontravam fora do eixo temático deste estudo.

$\mathrm{Na}$ etapa inicial da pesquisa foram encontrados 26 artigos na base de dados LILACS, 19 estudos foram excluídos, sendo 01 por repetição, 02 por fugirem da temática e 16 por apresentarem idioma, ano de publicação e disponibilidade divergente do estabelecido, restando 09 artigos. Na base de dados SciELO foram encontrados 07 artigos, desses, 02 foram excluídos após leitura de títulos e resumos, resultando em um total de 05 artigos. Na MEDLINE não obteve resultados.

Para a organização das 14 produções científicas selecionadas foi construído um quadro sinóptico contendo: ano de publicação, autor, periódico, método e principais conclusões.

A partir dos artigos encontrados a analise se deu por meio da construção de duas categorias temáticas: 1) Integralidade do Cuidado da Criança; 2) Vigilância em Saúde da Criança e o preenchimento do cartão do infante. 
RESULTADOS

O quadro a seguir retrata a descrição dos artigos selecionados. Pode-se observar o ano de publicação, o au- tor, o periódico, o método e principais conclusões. A maioria dos artigos são do ano de 2012, com predominância de pesquisas qualitativas.
DISCUSSÃO

Integralidade do cuidado da criança

Compreende-se a integralidade como um conjunto articulado e contínuo de

\section{QUADRO I - Panorama geral dos artigos selecionados, Crato/CE, 2018.}

Título

Objetivo

Autor/ano

Periódico

Método

Para uma efetiva assistência à criança na atenção básica, deve-se

Percepção materna do estado nutricional de seus filhos menores de três anos.

Avaliar a percepção materna do estado nutricional do filho.
Duarte, et al Rev. Esc Estudo (2016) Enferm USP transversal considerar a percepção materna e auxiliar as mães na identificação do estado nutricional do filho nas consultas de puericultura e acompanhamento do crescimento.

Mortalidade entre menores de um ano: análise dos casos após alta das maternidades.
Analisar as mortes infantis após alta das maternidades ocorridas entre 2000 e 2013.
Santos, et al Rev. Esc Quantita(2016) Enferm USP tivo
A quase totalidade das crianças nasceu em boas condições de vitalidade, apresentou agravos por doenças potencialmente preveníveis que culminaram no óbito.
Análise da situação vacinal de crianças pré-escolares em Teresina (PI).

\section{Analisar a situação vacinal de crianças pré escolares em} Teresina - PI.
Fernandes, Rev. BRAS Estudo

et al (2015) EPIDEMIOL seccional
A garantia e fortalecimento das ações da atenção básica são ferramentas imprescindíveis para reduzir atrasos e não vacinação.
Atenção à Saúde da Criança: prática de enfermeiros da saúde da família.
Analisar a prática, facilidades e dificuldades do enfermeiros na operacionalização do Programa de Acompanhamento do Desenvolvimento e Crescimento da Criança.
A maior facilidade para a pratica do cuidado da criança é o vínculo

Sousa, et al. (2013)

REME Qualitativo entre a equipe $e$ a mulher durante 0 pré-natal e a maior dificuldade são fatores culturais da mãe e da família quanto à adesão às orientações em cuidados essenciais à criança.
Consulta de puericultura realizada pelo enfermeiro na estratégia saúde da família.
Identificar ações implementadas pelo enfermeiro durante as consultas de puericultura em Unidades de Saúde da família.
Oliveira, et

al. (2013)
Averiguou-se a necessidade de capacitação dos enfermeiros para aperfeiçoamento do cuidado, visando contribuir com a melhoria da qualidade da assistência de enfermagem voltada a promoção da saúde da criança durante as consultas de puericultura.
Puericultura na atenção primária à saúde: atuação do enfermeiro.
Conhecer aspectos relacionados à atuação do enfermeiro na puericultura.
Vieira, et al. Cogitare

(2012) Enferm.

As discussões ensejaram reflexões Qualitativo a respeito da prática da enfermagem na puericultura. 
Descrever o quotidiano de enfermeiras na consulta em Quotidiano de enfermeiras: consulta em puericultura. puericultura e identificar fatores que influenciam a realização dessa consulta.
No cotidiano da puericultura, a enfermeira estabelece ações e Ribeiro, et Rev. Enferm Qualitativo interações coma criança e a família, al. (2014) UERJ Qualitativo promovendo a saúde, o cuidado efetivo e o respeito às diversidades ideológicas, sociais e culturais.
Descrever o perfil da população menor de um ano atendida na Puericultura em Enfermagem: consulta de enfermagem em perfil e principais problemas Puericultura e avaliar a associaencontrados em crianças menores de um ano. ção entre situação nutricional e presença de candidíase oral, candidíase perineal e dermatite de contato irritativa de fraldas.
0 estudo aponta a necessidade de sensibilizar a população a respeito Gauterio, et Rev. Bras Descritivo da importância da puericultura para al. (2012) Enferm Descritivo da importância da puença de agravos e promoção da saúde das crianças.
Conhecer as percepções dos profissionais de saúde da Atenção Básica sobre o seguimento do bebê pré-termo e/ou de baixo peso e sua família e a interface com a terceira etapa do Método Canguru.
Seguimento do bebê na atenção básica: interface com a terceira etapa do método canguru.
Analisar os diagnósticos de enfermagem constantes de

Mapeamento cruzado de diagnósticos de enfermagem em puericultura utilizando a CIPE. prontuários de crianças de $0 \mathrm{a}$ 36 meses de idade atendidas em consulta de enfermagem em puericultura. Luciano, et Rev. Esc Quantita- enfermagem, contribuindo para o al. (2014) Enferm USP tivo cuidado ao paciente e facilitando a comunicação entre os enfermeiros e outros profissionais de saúde.
Qualificando o cuidado à criança na Atenção Primária de Saúde.
Compreender modos de cuidar e de cuidado à criança na Atenção Primária de Saúde, com base no Grounded Theory. $\begin{array}{cc}\text { Sousa, et al. } & \text { Rev. Bras } \\ \text { (2012) } & \text { Enferm }\end{array}$
Qualitativo

0 cuidado se constrói a partir de esforços, colaboração, comunicação, complementaridade e no fazer/agir de profissionais, famílias, serviços e gestores.
Vigilância do crescimento infantil: conhecimento e práticas de enfermeiros da atenção primária à saúde.
Verificar os conhecimentos e práticas de enfermeiros da Estratégia Saúde da Família quanto à vigilância do crescimento de lactentes nas consultas de puericultura e informações maternas.

Reichert, et
al (2012) $\quad \begin{gathered}\text { Quantita- } \\ \text { tiva }\end{gathered}$

Identificou-se a necessidade de atualização dos enfermeiros sobre conteúdos relacionados à saúde da criança, para realizarem a vigilância do crescimento infantil de forma integral.
Registros de puericultura na atenção básica: estudo descritivo.
Investigar nos registros de prontuários como a puericultura é realizada pelas equipes da Estratégia Saúde da Família.
A implementação de uma ficha padrão para o registro das consultas de puericultura deve minimizar a subnotificação das atividades de acompanhamento às crianças. 


\author{
Acolhimento à adolescente \\ na consulta de enfermagem \\ - estudo qualitativo.
}

Compreender a relação do enfermeiro com a mãe adolescente na consulta de puericultura sob o enfoque do acolhimento.

\section{Brasil, et al Online braz j (2012)}

A escuta é uma ferramenta que o enfermeiro utiliza para realizar o acolhimento da mãe adolescente.

FONTE: Elaborado pelos autores, 2018.

ações e serviços preventivos, curativos, individuais e coletivos nos diferentes níveis de complexidade do sistema de saúde ${ }^{(10)}$.

Desde a década de 80 no Brasil, as políticas públicas voltadas para a saúde da criança sofreram aperfeiçoamentos. Implementou-se no país os programas voltados aos infantis, sendo eles o Programa Nacional de Imunização (PNI), o Programa de Assistência Integral à Saúde da Criança (PAISC) e a ESF, que trabalham de forma interdependentes com objetivos em comum, que é a redução da mortalidade infantil ${ }^{(11,12,13)}$.

Nesse contexto, as enfermeiras da ESF são inseridos como atores significativos na realização da puericultura, pois é de sua competência prestar assistência à criança por meio de consultas de puericultura, visitas domiciliares e busca ativa, além das intervenções educativas em grupo, que podem contribuir para desmistificar conceitos, promover práticas alimentares saudáveis e prevenir agravos ${ }^{(6,14)}$.

Através da consulta em puericultura à enfermeira acompanha o crescimento e desenvolvimento da criança, promove aleitamento materno, orienta alimentação por ocasião do desmame, faz controle das doenças imunopreveníveis pela vacinação, controle das doenças que comumente acometem os infantis no primeiro ano de vida, soluciona intercorrências e orienta mãe/cuidador sobre cuidados de saúde da criança. E a qualidade destas consultas está diretamente relacionado à disposição de uma rede de assistência que garantem o cuidado continuado ${ }^{(14,15,16)}$.

A educação em saúde deve sempre está presente em todo encontro com a criança, familiar e ou comunidade. Nesses momentos, o profissional de enfermagem deve utilizar para avaliar a criança, família e o meio no qual a criança está inserida oferecendo um cuidado integral e equânime. É preciso envolver os pais ou responsáveis no cuidado dos infantes, visto que, a percepção dos cuidadores são o gatilho para a busca de cuidado à saúde. Mas ainda há necessidade de alertar a população sobre a importância da puericultura para prevenir agravo na infância e os agravos que poderão repercutir na vida adulta ${ }^{(6,14,17)}$.

Torna-se necessário que os profissionais compreendam os múltiplos fatores sociais, econômicos e culturais que interferem no processo de saúde e doença e sua função como agentes de mudança da sociedade, procurando alternativas resolutivas dentro da abrangência de sua governabilidade. É importante ressaltar que a resistência dos pais e cuidadores em aderir aos programas de cuidados à saúde da criança estão ligados a crenças, cultura dos mais velhos, a baixa valorização do programa, falta de Agentes Comunitários de Saúde (ACS) considerando áreas descobertas, além da falta de material ${ }^{(15,17,8,12)}$.

No tocante a sistematização de enfermagem, essa permite a identificação de problemas, interpretação e organização de condutas no âmbito do exercício profissional. Dessa forma, a consulta de enfermagem tona-se um local de apoio, discussão e compartilhamento, permitindo o fortalecimento de vínculos afetivos na relação e proporcionando um cuidado de si e do outro, sendo também necessário um protocolo referente às consultas de puericultura para nortear as ações desses profissionais de saúde ${ }^{(16,11,15,17)}$.

Ainda é referido a importância da visita domiciliar (VD) para a continuidade do cuidado iniciado no pré-natal. Porém, há varias dificuldade encontradas pelas equipes de saúde, como falta de meios de lo- comoção da equipe, sobrecarga de trabaIho da enfermeira, entre outros pontos ${ }^{(19)}$.

Vigilância em saúde da criança e o preenchimento do cartão do infante

O papel da enfermeira na vigilância da saúde da criança apresenta grande importância, especialmente nos serviços de atenção primária à saúde, no sentido de viabilizar o melhor acompanhamento do crescimento e desenvolvimento, visto ser essa uma ação fundamental para se obter melhor qualidade de vida para a população infantil (20).

Um dos elementos para o programa de vigilância em saúde da criança é conhecer a cobertura vacinal em menores de um ano, na medida em que permite visualizar o aumento do número de indivíduos susceptíveis a falta de adesão na população, bem como acompanhar até que ponto os programas de imunização em massa estão atuando como barreira efetiva para a interrupção da transmissão das doenças imunopreviníveis ${ }^{(21)}$.

$\mathrm{O}$ crescimento e desenvolvimento infantil é considerado um dos melhores indicadores de saúde da criança, em função de sua estreita dependência de fatores econômicos, culturais e sociais relacionados à: tipo de alimentação em quantidade e qualidade; ocorrências e tipos de doenças; cuidados de saúde e vacinação; afetividade da família com a criança; condições de vida, destacando-se a moradia e o saneamento básico; e acesso aos serviços de saúde. Portanto, o acompanhamento adequado do crescimento infantil é reconhecido e recomendado como uma importante ação de saúde, sendo decisivo para a vigilância em saúde ${ }^{(17,12,16,18)}$.

Entretanto, a vigilância na saúde da 
criança na puericultura torna-se fragilizada a partir do momento em que o profissional de saúde não consegue usar adequadamente a caderneta da criança, como evidenciado em estudos realizados no Brasil e também pela Organização Mundial da Saúde envolvendo 178 países, ao demonstrar que $80 \%$ dos profissionais tinham dificuldades no uso do cartão de acompanhamento de crescimento infantil, em que os maiores problemas eram de natureza conceitual e operacional ${ }^{(20)}$.

O acompanhamento do crescimento e desenvolvimento é essencial para uma avaliação integral à saúde da criança. $\mathrm{O}$ acompanhamento envolve o registro no Cartão da Criança e no prontuário a avaliação de peso, altura, desenvolvimento, vacinação e intercorrências, o estado nutricional, bem como orientações à mãe/ família/cuidador sobre os cuidados com a criança em todo atendimento ${ }^{(13,14,17)}$.

$\mathrm{O}$ registro completo e correto das informações colhidas durante as consultas de puericultura é importante instrumento de comunicação, vigilância e promoção da saúde infantil. Tendo em vista que constitui meio de comunicação para o (re)planejamento, continuidade e avaliação dos serviços prestados aos clientes $^{(20)}$.

O prontuário do infante deve conter informações desde o pré-natal até o momento a consulta. Entretanto, estudos apontam que existe um sub-registro concernente as informações do atendimento, que consequentemente dificulta a continuidade da assistência por outros profissionais da saúde por não conterem informações substanciais ${ }^{(8,21)}$.

Toda esta organização de informações e documentos favorece um controle das ações realizadas no sentido de perceber sua eficácia, além de pautar uma prática que atenda às necessidades da criança e sua família.

\section{CONCLUSÃO}

Destaca-se a resistência dos pais e cuidados na adesão aos programas de cuidados à saúde da criança, como também a falta de transporte para o profissional realizar a visita domiciliar, a sobrecarga de trabalho da enfermeira e a falta de $\mathrm{ACS}$, que consequentemente deixa áreas descobertas na abrangência da ESF.

A dificuldade do preenchimento e da operacionalização do Cartão da Criança é um problema constatado a nível mundial pela Organização Mundial da Saúde, fragilizando a vigilância em saúde do infante. Outro fator que fragiliza a vigilância da saúde da criança é o sub-registro no prontuário dos atendimentos realizados.

O estudo limita-se por não ter abrangido todas as bases de dados existentes e por ter feito recorte temporal dos últimos cinco anos. Sugere-se que outras pesquisas sejam realizadas na perspectiva de captar estudos internacionais.

\section{Referências}

1. Mamede FV, prudencio PS. Programs and public policy contributions for the improvement of maternal health. Rev Gaúcha Enferm. 2015; 36(spe): 262-266. 2. Brasil. Ministério da Saúde. Departamento de Informática do SUS - DATASUS. Instituto Brasileiro de Geografia e Estatística. Projeção da População do Brasil - 2013. Available from: http://brasilemsintese.ibge.gov.br/populacao/taxas-de-mortalidade-infantil.html

4. Brasil. Ministério da Saúde. Secretaria de Políticas de Saúde. Departamento de Atenção Básica. Saúde da criança: acompanhamento do crescimento e desenvolvimento infantil / Ministério da Saúde. Secretaria de Políticas de Saúde. Brasília: Ministério da Saúde, 2002.

5. Andrade RD, Santos JS, Pina JC, Silva MAI, Mello DF. A puericultura como momento de defesa do direito à saúde da criança. Cienc Cuid Saude. 2013 Out/ Dez; 12(4): 719-727.

6. Sousa FGM, Erdmann AL. Qualificando o cuidado à criança na Atenção Primária de Saúde. Rev Bras Enferm. 2012 set-out; 65(5): 795-802.

7. Brasil. Ministério da Saúde. Secretaria de Atenção à Saúde. Departamento de Atenção Básica. Saúde da criança: crescimento e desenvolvimento (Cadernos de Atenção Básica, $n^{\circ}$ 33) / Ministério da Saúde. Secretaria de Atenção à Saúde. Departamento de Atenção. Básica. - Brasília : Ministério da Saúde, 2012.

8. Oliveira FFS, Oliveira ASS, Lima LHO, Marques MB, Felipe GF, Sena IVO. Consulta de puericultura realizada pelo enfermeiro na estratégia saúde da família. Ver. Rene. 2013; 14(4): 694-703.

9. Mendes KDS, Silveira RCCP, Galvão CM. Revisão integrativa: método de pesquisa para a incorporação de evidências na saúde e na enfermagem. Texto Contexto Enferm. 2008; 17(4): 758-764.

10. Fracolli LA, et al. Conceito e prática da integralidade na Atenção Básica: a percepção das enfermeiras. Revista da Escola de Enfermagem da USP, v. 45, n. 5, p. 1135-1141, 2011.

11. Santos EP, Ferrari RAP, Bertolozzi MR, Cardelli AAM, Godoy CB, Genovesi FF. Mortality among children under the age of one: analysis of cases after discharge from maternity. Rev Esc Enferm USP · 2016;50(3):390-398.

12. Souza, R. S.; Ferrari, RAP, Santos TFM, Tacla MTGM. Atenção à Saúde da Criança: prática de enfermeiros da saúde da família. Rev Min Enferm. 2013 abr/ jun; 17(2): 331-339

13. Fernandes ACN, Gomes KRO, Araújo TME, Moreira-Araújo RSR. Análise da situação vacinal de crianças pré-escolares em Teresina (PI). Rev bras epidemiol out-dez 2015; 18(4): 870-882.

14. Duarte LS, Fujimori E, Toriyama ATM, Palombo CNT, Miranda PPL, Borges ALV. Maternal perception of their child's nutritional status at less than three years old. Rev Esc Enferm USP. 2016;50(5):771-778.

15. Gauterio DP, Irala DA, Cezar-Vaz MR. Puericultura em Enfermagem: perfil e principais problemas encontrados em crianças menores de um ano. Rev Bras Enferm, Brasília 2012 mai-jun; 65(3): 508-13.

16. Ribeiro SP, Oliveira DS, Fernandes SLSA, Felzemburgh RDM, Camargo CL. 0 quotidiano de enfermeiras na consulta em puericultura. Rev enferm UERJ, Rio de Janeiro, $2014 \mathrm{jan} / \mathrm{fev}$; 22(1):89-95.

17. Vieira VCL, Fernandes CA, Demitto MO, Bercini LO, Scochi MJ, Marcon SS Puericultura na atenção primária à saúde: atuação do enfermeiro. Cogitare Enfer. 2012 Jan/Mar; 17(1): 119-125.

18. Luciano TS, Nóbrega MML, Saparolli ECL, Barros ALBL. Mapeamento cruzado de diagnósticos de enfermagem em puericultura utilizando a Classificação Internacional de Práticas de Enfermagem. Rev Esc Enferm USP 2014; 48(2):250-6. 19. Airesa LCP, Santosb EKA, Costab R, Borckc M, Custódioc ZAO. Seguimento do bebê na atenção básica: interface com a terceira etapa do método canguru. Rev Gaúcha Enferm. 2015;36(esp):224-32.

20. Reichert APS, Almeida AB, Souza LC, Silva MEA, Collet N. Vigilância do crescimento infantil: conhecimento e práticas de enfermeiros da atenção primária à saúde. Rev Rene. 2012; 13(1):114-26.

21. Barboza CL, Barreto MS, Marcon SS. Registros de puericultura na atenção básica: estudo descritivo. Online braz j nurs [periodic online]. 2012 Aug; 11(2): 359-75. 